\title{
Regional Anaesthesia for Arterio-Venous fistula in End Stage Renal Failure patients
}

\author{
Chetan Srinath, Leeds Teaching Hospitals NHS Trust
}

\section{Introduction}

Approximately $4.5 \%$ of the people in UK have chronic kidney disease. End stage renal failure (ESRF) creates a huge health burden. ESRF patients end up having dialysis if they don't get a transplant in time. These patients may opt for haemodialysis or peritoneal dialysis. Patients who choose to have haemodialysis will need arterio-venous (AV) fistula. The most common are radiocephalic or brachio-cephalic fistula. These fistulae needs a maturation time of 4 to 6 weeks. AVF have high failure rate of 30$40 \%$ which could be due to failed maturation or thrombosis. The choice of anaesthetic technique for formation of these fistulae can vary. This can be done under local or regional anaesthesia (RA) or general anaesthesia. This group of patients have several comorbidities and may pose additional risks when exposed to general anaesthesia. Local anaesthesia infiltration during surgery can result in vasospasm and local oedema. RA in the form of brachial plexus blocks are useful in the formation of $\mathrm{AV}$ fistula. RA has a good safety profile as it reduces the exposure to additional anaesthetic drugs. RA provides excellent anaesthesia and good postoperative analgesia and can be done as a day case technique.

\section{Methods}

We studied 10 patients in ESRF coming for the formation of primary AV fistula at St James University Hospital, Leeds. All patients were consented pre-procedure. Patients coming for redofistula, brachial vein transposition and $\mathrm{AV}$ fistula graft were excluded. Lignocaine $2 \%$ with adrenaline was used for the brachial plexus block. Cephalic vein and brachial artery sizes were measured with ultrasound at the elbow pre block and 20 minutes post block by the same surgeon.

\section{Results}

RA resulted in vasodilatation of the vessels. The average increase in size of cephalic vein was $14.8 \%$ with the maximum increase up to $55 \%$. The average increase in size of the brachial artery was $6.4 \%$. There was more increase in diameter of cephalic vein than brachial artery.

\section{Conclusion}

RA provides dense and prolonged sensory and motor blockade. The sympathectomy results in decreased peripheral vascular resistance resulting in vasodilatation and increased blood flow velocity in the intraoperative and postoperative period. This higher flow rates help to maintain patency by preventing thrombosis and potentially improving outcome. These effects of RA can assist in preventing failure, increasing the success rates and early maturation of fistula.

RA caused vasodilation can provide surgical site flexibility by formation of fistula peripherally and save the proximal areas for the future. RA with good motor block and vasodilatation provides an ideal condition for the surgeon during the operation. RA is also associated with shorter length of stay and lower risk of re-operations. With RA, patients can be discharged home the same day resulting in higher patient satisfaction and reduced health care costs.

\section{References}

1. Anesthesia for vascular access surgery. Shemesh D, Raikhinstein Y, Orkin D, Goldin I, Olsha O. J Vasc Access. 2014;15 Suppl 7:S3844.

2. Regional Anesthesia for Arteriovenous Fistula Surgery May Reduce Hospital Length of Stay and Reoperation Rates. Cole NM, Vlassakov K, Brovman EY, Heydarpour M, Urman RD. Vasc Endovascular Surg. 2018 Aug;52(6):418-426

\section{Cephalic vein}

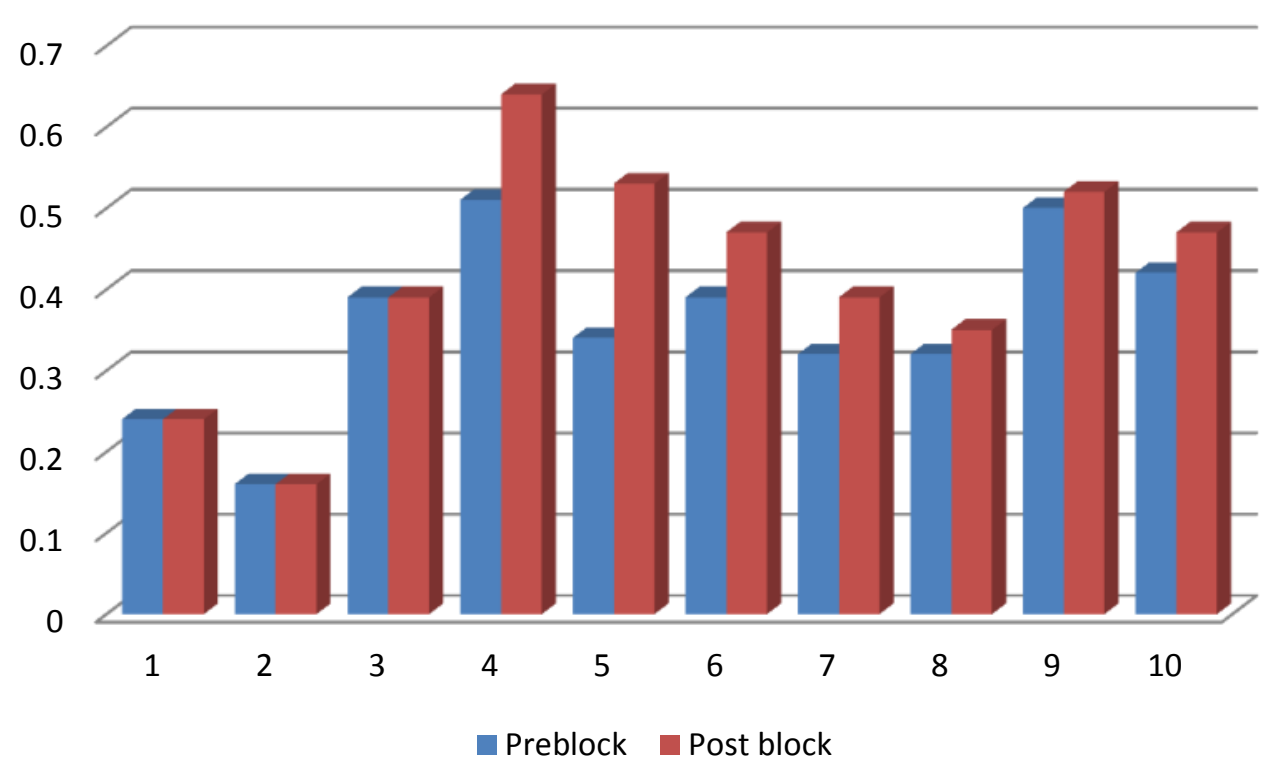

Brachial Artery

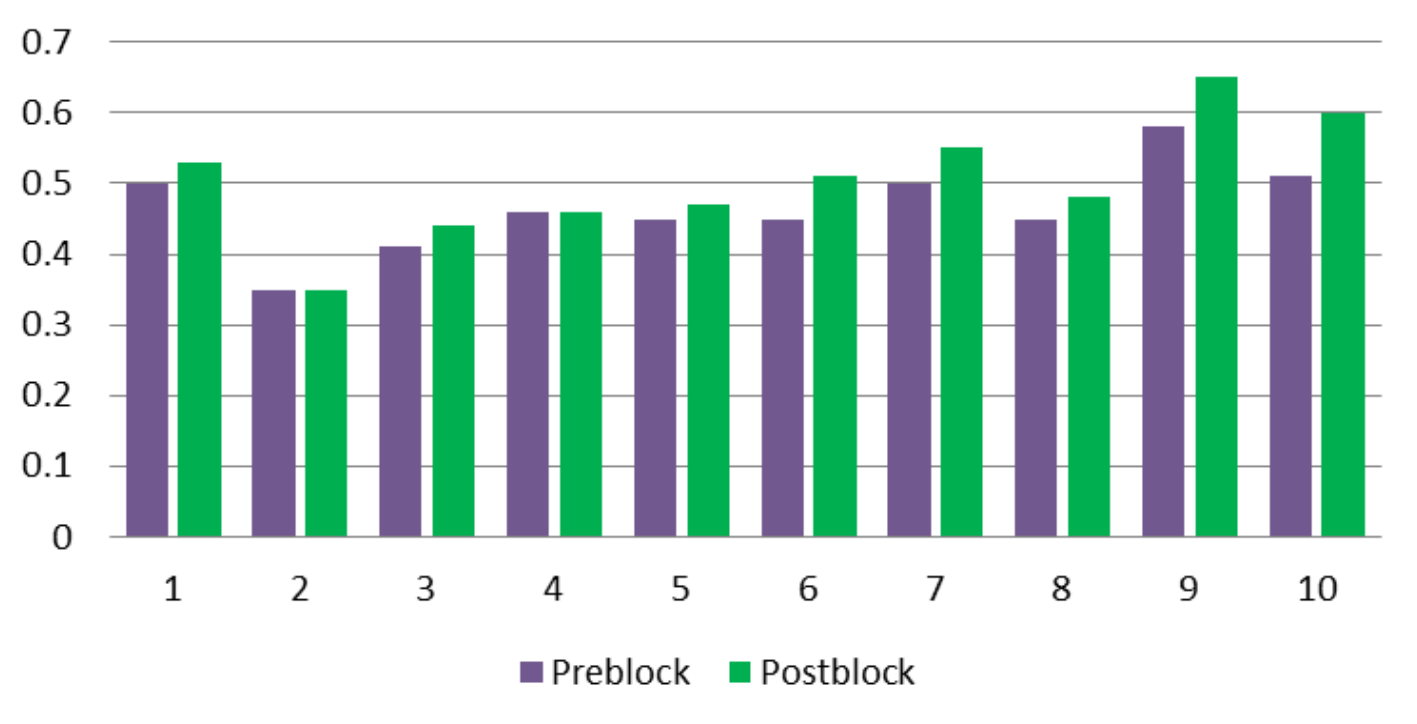

